\title{
Verification of Neutron Data for Main Reactor Materials from RUSFOND Library based on Integral Experiments
}

\author{
V. N. Koshcheev ${ }^{1, \mathrm{a}}$, G. N. Manturov ${ }^{1}$, M. N. Nikolaev ${ }^{1}$, A. M. Tsibouliya ${ }^{1}$ \\ ${ }^{1}$ IPPE, Obninsk, Russia 249033
}

\begin{abstract}
In this work the modern state of the library of evaluated nuclear data files RUSFOND for the main reactor materials, U235, U238, Pu239, Fe, Cr, Ni, Na, Pb, etc., is given. Calculations are performed and comparison with experimental data is done for the following characteristics: (i) Removal cross-sections under the threshold of fission of U-238 etc... (ii) Average cross-sections with different standard neutron fission spectra; (iii) Criticality of fast uranium and plutonium systems. Calculations are performed using continuous energy cross-sections and a Monte-Carlo code.
\end{abstract}

\section{Introduction}

National neutron data libraries were recently modified in USA, Europe Japan, China and Russia. These data libraries are now being tested for further use in applications. The neutron data were mainly tested using criticality calculations for the benchmarks selected by a special procedure from the ICSBEP Handbook. Other tests included 1) calculations of average cross sections on standard and some specially selected spectra, and 2) calculations of the cross sections of neutron removal under the thresholds of detector reactions such as (n,fis)U-238, (n,fis)Np-237, (n,p)Al-27, etc. These results are presented in this work.

\section{Neutron data libraries}

The following neutron data libraries were used: the Russian library ROSFOND presented in 2008 and corrected in 2010 [1]; the Japanese library JENDL-4.0 presented in 2011 [2]; the US library ENDF/B-VII.1 presented in 2011 [3]; the European library JEFF-3.1.1 presented in 2009 [4]. The main reactor nuclides were selected as follows:

- Fuel materials - U-235, U-238, Pu-239;

- $\quad$ Structural materials - Fe, $\mathrm{Cr}, \mathrm{Ni}$;

- Coolant materials - $\mathrm{Na}, \mathrm{Pb}$.

\footnotetext{
a e-mail: abbn@ippe.ru
} 


\section{Benchmarks used}

The Mughabghab evaluation of resonance integrals (the spectrum of $\sim 1 / \mathrm{E}$ ) was used as a reference [5]. Average cross sections calculated on the U-235 and Cf-252 fission spectra as well as removal cross sections from the EXFOR Library were taken as reference values [6]. The input data and corresponding benchmark criticalities for selected benchmarks were taken from ICSBEP Handbook [7]. The criteria for selecting benchmarks were 1) simplicity of benchmark models, 2) representativeness with respect to the $\mathrm{H} /$ fuel ratio (from low to high values), 3) description completeness, and others [8]. 62 models with the HEU type fuel, 49 models with the LEU type fuel, and 102 models with the Pu fuel were selected. Neutron data for U-235, U-238 and Pu-239 were preliminary tested in criticality calculations of the Pruvost test models [9].

\section{Results}

The comparison of the results on resonance integrals (the spectrum of $\sim 1 / \mathrm{E}$ ) for $\mathrm{Na}$ and $\mathrm{Pb}$ are presented in Table 1, and for U-235, U-238 and Pu-239 in Table 2. In Tables 1-2 the following notation is used: "LMFBR" stands for the neutron spectrum of a typical sodium-cooled fast breeder reactor, "U235" stands for the fission spectrum of U-235. 30-group cross sections (the energy structure accepted in the ICSBEP Handbook is applied) were used in verification. They were prepared by NJOY [10]. The experimental values of the removal cross sections presented in Table 3 were taken from Ref. [11]. The comparison of the results of criticality calculations of the Pruvost test models was done on the next stage of verification. The calculations were performed by MCNP5 [12]. Data for generating input files for MCNP were taken from Ref. [9]. The dependence of the calculation values of criticality and corresponding $\mathrm{C} / \mathrm{E}$ ratio values on the content of fuel nuclides is presented in Fig.1 for the case of homogeneous mixture of HEU and water with water reflector, and for the case of homogeneous mixture of PU and water with water reflector. The next stage of verification was done on the base of criticality calculations for the benchmark set selected from the ICSBEP Handbook. The C/E ratio for the sodium benchmark (MCF004) is presented in Fig. 2. Similar results for the set of the lead benchmarks are presented in Fig. 2 both. The C/E ratios for the HEU type benchmarks are presented in Fig. 3, for the LEU type benchmarks in Fig. 4, and for the PU type benchmarks in Fig. 5. Criticality calculations were performed by MCNP5. The reference (experimental) values were taken from the ICSBEP Handbook. For convenience, all final results are collected in Table 4.

Table 1. The comparison of the average cross sections for coolant nuclide.

\begin{tabular}{|c|c|c|c|c|c|c|c|}
\hline $\mathrm{Na}$ & capture & total & inel & $\mathrm{Pb}$ & capture & total & inel \\
\hline$\varphi(L M F B R)$ & & & & $\varphi(L M F B R)$ & & & \\
\hline RF2010 & 1.000 & 1.000 & 1.000 & RF2010 & 1.000 & 1.000 & 1.000 \\
\hline E-VII.1 & 1.000 & 1.000 & 1.000 & E-VII.1 & 1.000 & 1.000 & 0.992 \\
\hline JEFF-3.1.2 & 0.931 & 1.037 & 0.814 & JEFF-3.1.2 & 1.000 & 1.000 & 1.000 \\
\hline JENDL-4.0 & 0.946 & 1.029 & 1.083 & JENDL-4.0 & 1.104 & 1.019 & 1.005 \\
\hline$\varphi(U-235)$ & & & & $\varphi(U-235)$ & & & \\
\hline RF2010 & 1.000 & 1.000 & 1.000 & RF2010 & 1.000 & 1.000 & 1.000 \\
\hline E-VII.1 & 1.001 & 1.000 & 1.000 & E-VII.1 & 1.001 & 1.000 & 0.990 \\
\hline JEFF-3.1.2 & 0.977 & 1.062 & 0.732 & JEFF-3.1.2 & 1.000 & 1.000 & 1.000 \\
\hline JENDL-4.0 & 1.039 & 1.001 & 1.016 & JENDL-4.0 & 1.306 & 0.991 & 1.006 \\
\hline
\end{tabular}


Table 2. The comparison of the average cross sections for fission nuclide.

\begin{tabular}{|c|c|c|c|c|c|c|c|c|c|}
\hline U-235 & capture & inel & fission & nu-bar & U-238 & capture & inel & fission & nu-bar \\
\hline$\varphi(L M F B R)$ & & & & & $\varphi(L M F B R)$ & & & & \\
\hline RF2010 & 1.000 & 1.000 & 1.000 & 1.000 & RF2010 & 1.000 & 1.000 & 1.000 & 1.000 \\
\hline E-VII.1 & 1.000 & 1.008 & 0.994 & 1.001 & E-VII.1 & 1.000 & 1.052 & 1.000 & 0.993 \\
\hline JEFF-3.1.2 & 1.003 & 0.999 & 0.997 & 1.000 & JEFF-3.1.2 & 1.001 & 0.952 & 0.998 & 1.001 \\
\hline JENDL-4.0 & 0.956 & 0.998 & 0.984 & 1.000 & JENDL-4.0 & 0.999 & 0.990 & 0.999 & 0.994 \\
\hline $\operatorname{EXP} \varphi(U-235)$ & & & 1.000 & & $\operatorname{EXP} \varphi(U 235)$ & & & 1.000 & \\
\hline RF2010 & 1.000 & 1.000 & 1.006 & 1.000 & RF2010 & 1.000 & 1.000 & 0.985 & 1.000 \\
\hline E-VII.1 & 1.001 & 1.023 & 1.003 & 1.002 & E-VII.1 & 1.000 & 1.013 & 0.985 & 0.992 \\
\hline JEFF-3.1.2 & 1.000 & 0.999 & 0.998 & 1.000 & JEFF-3.1.2 & 0.988 & 1.028 & 0.980 & 1.000 \\
\hline JENDL-4.0 & 0.945 & 0.960 & 0.998 & 1.003 & JENDL-4.0 & 1.011 & 1.000 & 0.981 & 0.994 \\
\hline $\operatorname{EXP} \varphi(C f-252)$ & & & 1.000 & & $\overline{\operatorname{EXP}} \varphi(C f 252)$ & & & 1.000 & \\
\hline RF2010 & 1.000 & 1.000 & 0.994 & 1.000 & RF2010 & 1.000 & 1.000 & 0.947 & 1.000 \\
\hline E-VII.1 & 1.000 & 1.024 & 0.991 & 1.001 & E-VII.1 & 1.000 & 1.013 & 0.947 & 0.992 \\
\hline JEFF-3.1.2 & 1.000 & 0.999 & 0.986 & 1.000 & JEFF-3.1.2 & 0.988 & 1.030 & 0.941 & 1.000 \\
\hline JENDL-4.0 & 0.944 & 0.961 & 0.986 & 1.003 & JENDL-4.0 & 1.010 & 1.001 & 0.942 & 0.994 \\
\hline Pu-239 & capture & inel & fission & nu-bar & & & & & \\
\hline$\underline{\varphi(L M F B R)}$ & & & & & & & & & \\
\hline RF2010 & 1.000 & 1.000 & 1.000 & 1.000 & & & & & \\
\hline E-VII.1 & 0.984 & 1.341 & 1.006 & 1.001 & & & & & \\
\hline JEFF-3.1.2 & 1.000 & 1.000 & 1.000 & 1.000 & & & & & \\
\hline JENDL-4.0 & 0.979 & 1.249 & 1.009 & 1.003 & & & & & \\
\hline $\operatorname{EXP} \varphi(U-235)$ & & & 1.000 & & & & & & \\
\hline RF2010 & 1.000 & 1.000 & 0.976 & 1.000 & & & & & \\
\hline E-VII.1 & 0.755 & 1.185 & 0.975 & 1.001 & & & & & \\
\hline JEFF-3.1.2 & 1.000 & 1.000 & 0.976 & 1.000 & & & & & \\
\hline JENDL-4.0 & 0.987 & 1.174 & 0.979 & 0.997 & & & & & \\
\hline $\operatorname{EXP} \varphi(C f-252)$ & & & 1.000 & & & & & & \\
\hline RF2010 & 1.000 & 1.000 & 0.978 & 1.000 & & & & & \\
\hline ENDF/B-VII.1 & 0.759 & 1.182 & 0.977 & 1.001 & & & & & \\
\hline JEFF-3.1.2 & 1.000 & 1.000 & 0.978 & 1.000 & & & & & \\
\hline JENDL-4.0 & 0.986 & 1.171 & 0.981 & 0.997 & & & & & \\
\hline
\end{tabular}

Table 3. The comparison of the removal cross sections.

\begin{tabular}{|c|c|c|c|c|c|c|c|}
\hline $\mathrm{Na}$ & $(n, f) N p 237$ & $(n, f) U 238$ & $(n, p) A l 27$ & $\mathrm{~Pb}$ & $(n, f) N p 237$ & $(n, f) U 238$ & $(n, p) A l 27$ \\
\hline EXP & & 1.000 & & EXP & 1.000 & 1.000 & 1.000 \\
\hline RF2010 & 1.000 & 0.831 & 1.000 & RF2010 & 1.300 & 1.182 & 1.060 \\
\hline E-VII.1 & 1.000 & 0.831 & 0.999 & E-VII.1 & 1.251 & 1.145 & 1.036 \\
\hline JEFF-3.1.2 & 1.005 & 0.720 & 0.732 & JEFF-3.1.2 & 1.300 & 1.182 & 1.060 \\
\hline JENDL-4.0 & 1.113 & 0.851 & 0.999 & JENDL-4.0 & 1.298 & 1.188 & 1.067 \\
\hline U-235 & $(n, f) N p 237$ & $\overline{(2 n, f) U 238}$ & $\overline{(2 n, p) A l 27}$ & $\mathrm{U}-238$ & $(\bar{l}(n, f) N p 237$ & (n,f)U238 & $\overline{(n, p) A l 27}$ \\
\hline EXP & & 1.000 & & EXP & & 1.000 & \\
\hline RF2010 & 1.000 & 1.151 & 1.000 & RF2010 & 1.000 & 1.007 & 1.000 \\
\hline E-VII.1 & 1.009 & 1.180 & 1.058 & E-VII.1 & 1.002 & 1.007 & 1.000 \\
\hline JEFF-3.1.2 & 0.989 & 1.139 & 0.992 & JEFF-3.1.2 & 1.017 & 1.058 & 1.108 \\
\hline JENDL-4.0 & 0.975 & 1.169 & 1.045 & JENDL-4.0 & 0.963 & 0.994 & 1.072 \\
\hline Pu-239 & $\begin{array}{l}(n, f) N p 237 \\
\end{array}$ & $\overline{(2 n, f) U 238}$ & $\overline{(2 n, p) A l 27}$ & & & & \\
\hline EXP & & 1.000 & & & & & \\
\hline RF2010 & 1.000 & 0.819 & 1.000 & & & & \\
\hline E-VII.1 & 1.196 & 0.944 & 1.087 & & & & \\
\hline JEFF-3.1.2 & 1.000 & 0.819 & 1.000 & & & & \\
\hline JENDL-4.0 & 1.245 & 1.001 & 1.122 & & & & \\
\hline
\end{tabular}


Table 4. The average benchmark uncertainties and the results for $\mathrm{C} / \mathrm{E}-1$ for benchmark set (in pcm).

\begin{tabular}{||l|c|c|c|c|c|c||}
\hline $\begin{array}{c}\text { Benchmark } \\
\text { type }\end{array}$ & $\begin{array}{c}\text { Number of } \\
\text { benchmarks }\end{array}$ & $\begin{array}{c}\text { Average } \\
\text { benchmark } \\
\text { uncertainty }\end{array}$ & RF2010 & E-VII.1 & JEFF-3.1.1 & JENDL-4.0 \\
\hline \hline Cr capture & 1 & 180 & 904 & 766 & 874 & 1121 \\
\hline Fe capture & 6 & 44 & 133 & 222 & 252 & 114 \\
\hline Fe reflector* & 23 & 26 & -39 & 2 & -26 & -25 \\
\hline N capture & 1 & 40 & -118 & -64 & -115 & -153 \\
\hline $\boldsymbol{N}$ reflector & 6 & 26 & 2 & 30 & 1 & 4 \\
\hline Na & 1 & 15 & -23 & 29 & 41 & 57 \\
\hline $\boldsymbol{P b}$ & 10 & 20 & 6 & -46 & -7 & -3 \\
\hline $\boldsymbol{H E U}$ fast & 7 & 21 & -1 & 4 & -30 & -12 \\
\hline $\boldsymbol{H E U}$ thermal & 55 & 39 & -11 & -6 & -6 & 4 \\
\hline $\boldsymbol{L E U}$ fast & 14 & 35 & 1 & -20 & -51 & -71 \\
\hline $\boldsymbol{L E U}$ thermal & 35 & 26 & 9 & 6 & 11 & -7 \\
\hline $\boldsymbol{P U}$ fast & 8 & 21 & -14 & 4 & 0 & -11 \\
\hline $\boldsymbol{P U}$ thermal & 94 & 44 & 34 & 44 & 15 & 45 \\
\hline
\end{tabular}

* PMF42 (cases 3-15) and PMF45 (cases 2-4) are not t included.

\section{Discussion}

More information on $\mathrm{Cr}, \mathrm{Fe}$ and $\mathrm{Ni}$ is presented in the next poster. The impact of the choice of $\mathrm{Na}$ evaluation is presented in Fig. 2. It is seen that using neutron data for Na from RF2010 instead of JEFF-3.1.1 leads to the bias in calculation criticality by $\sim 0.5 \%$. The best agreement for the lead benchmarks is observed when using the JENDL-4.0 library (see Fig. 2). Calculation results for thermal systems (HEU, LEU and $\mathrm{Pu}$ ) based on different neutron libraries are in better agreement with each other than with corresponding benchmark values (see Fig.3-5). For the set of the fast benchmarks discrepancies between calculation and reference values can be, in some cases, as much as $1 \%$ and even greater (see Fig 6-7).

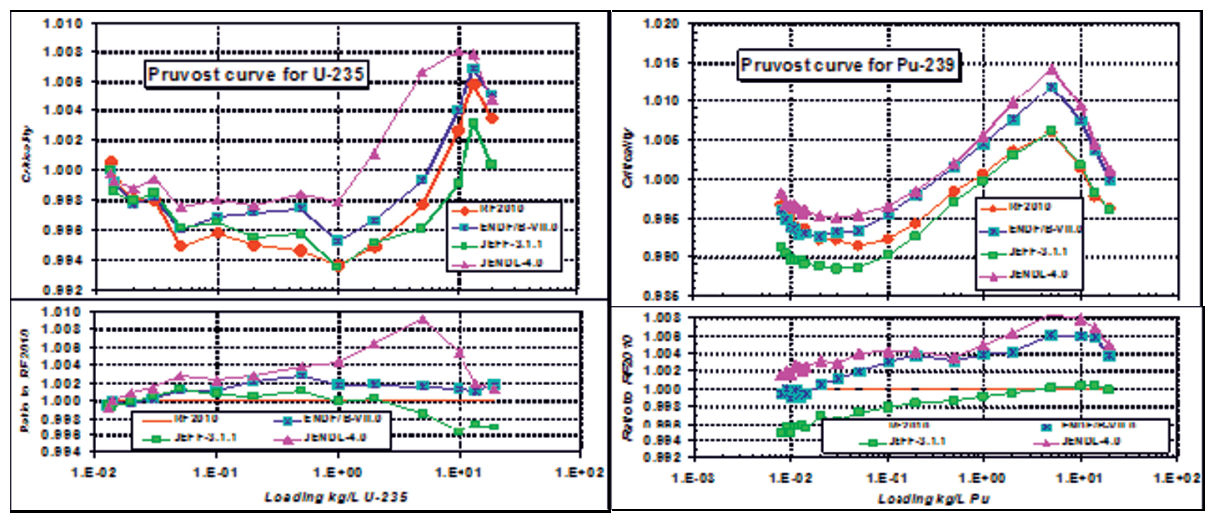

Fig. 1: Pruvost curves for U-235 and Pu-239 

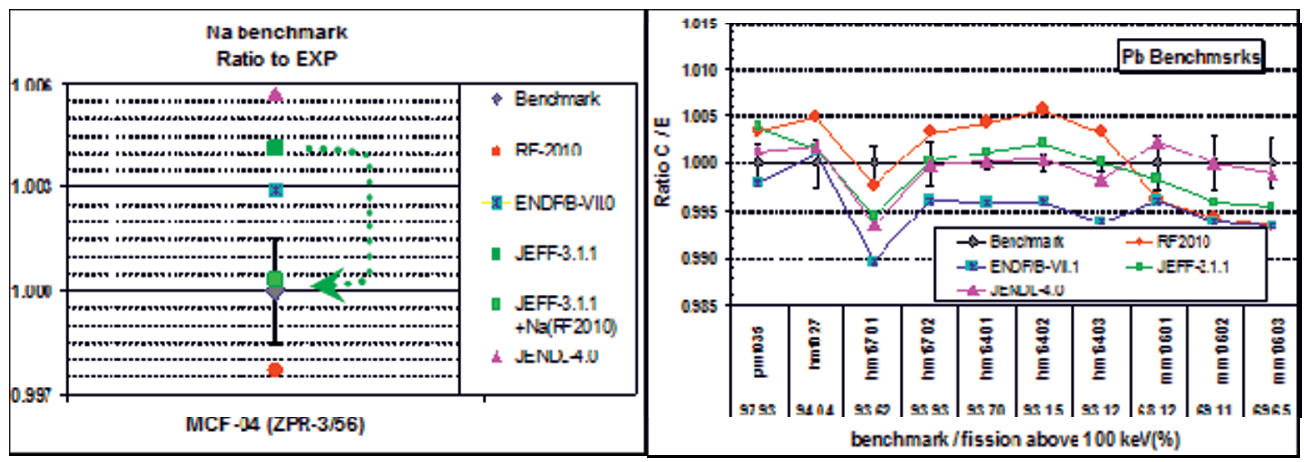

Fig. 2: $\mathrm{Na}$ and $\mathrm{Pb}$ benchmarks.

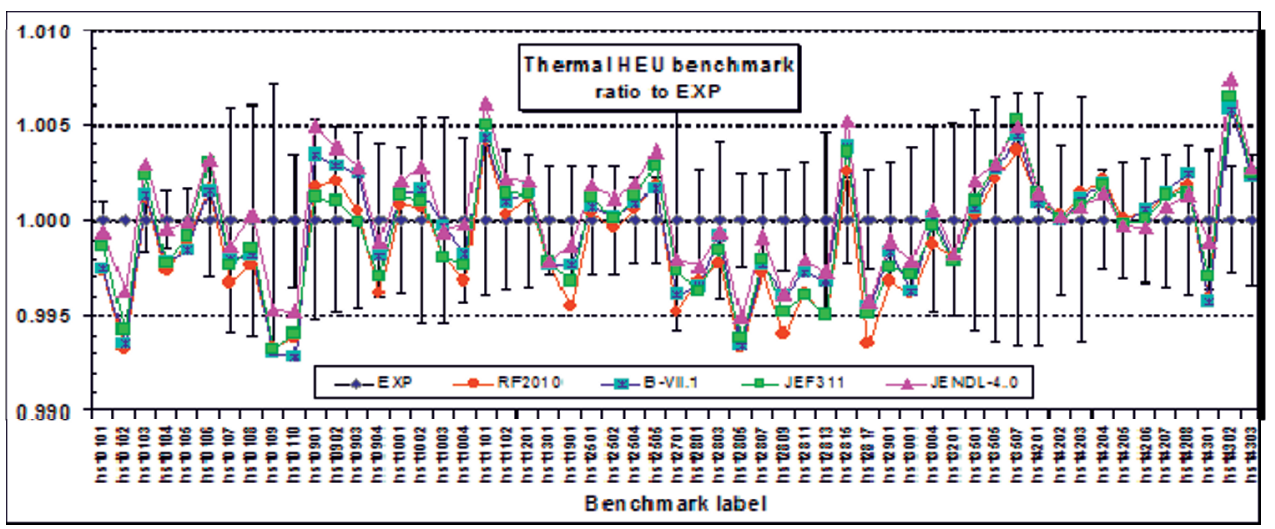

Fig. 3: Thermal HEU benchmarks.

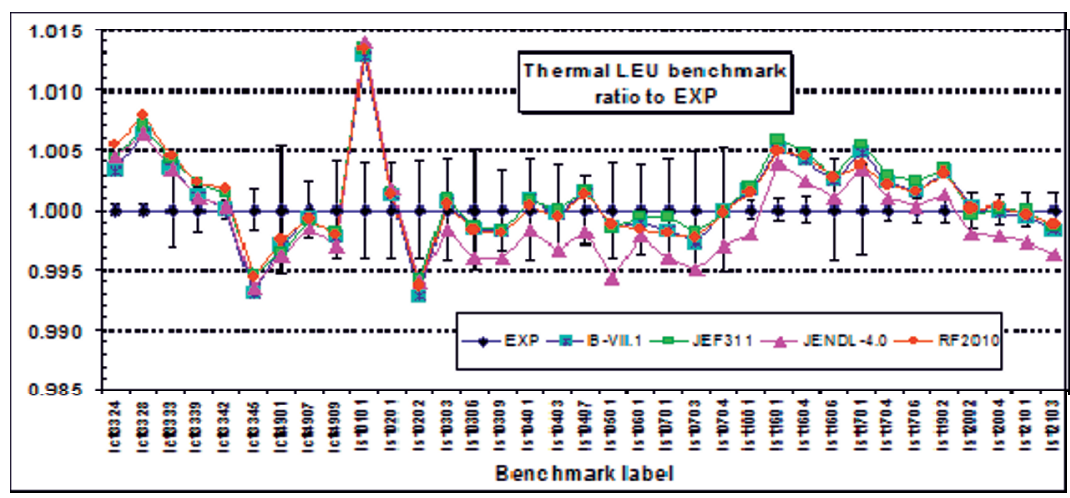

Fig. 4: Thermal LEU benchmarks. 


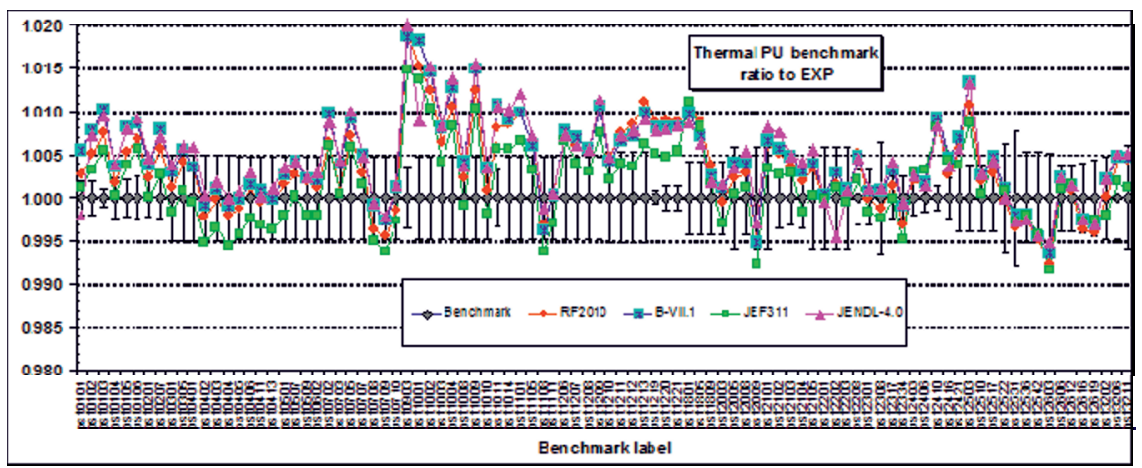

Fig. 5: Thermal Pu benchmarks.

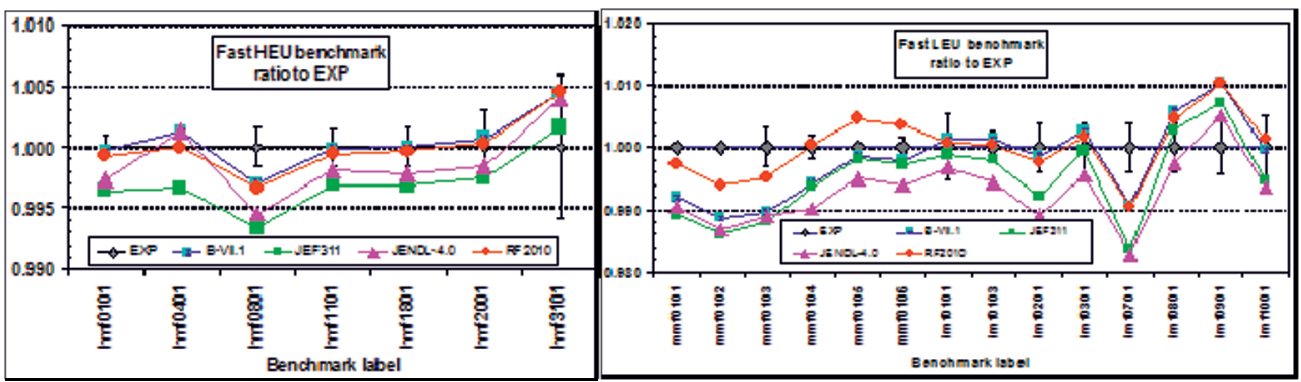

Fig. 6: Fast HEU and LEU benchmarks.

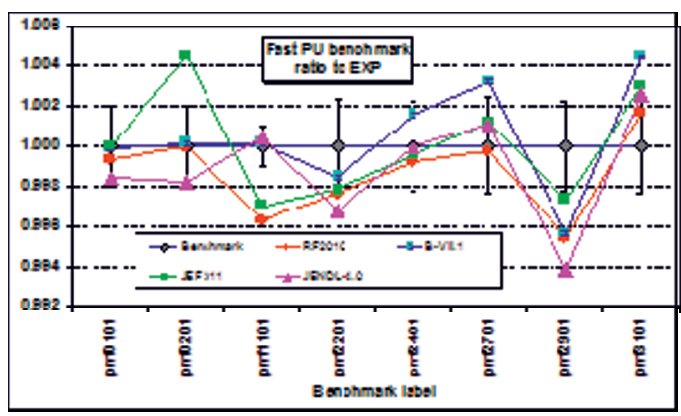

Fig. 7: Fast Pu benchmarks.

\section{Discussion}

More information on $\mathrm{Cr}, \mathrm{Fe}$ and $\mathrm{Ni}$ is presented in the next poster. The impact of the choice of $\mathrm{Na}$ evaluation is presented in Fig. 2. It is seen that using neutron data for Na from RF2010 instead of JEFF-3.1.1 leads to the bias in calculation criticality by $\sim 0.5 \%$. The best agreement for the lead benchmarks is observed when using the JENDL-4.0 library (see Fig. 2). Calculation results for thermal systems (HEU, LEU and Pu) based on different neutron libraries are in better agreement with each other than with corresponding benchmark values (see Figs 3-5). For the set of the fast benchmarks discrepancies between calculation and reference values can be, in some cases, as much as $1 \%$ and even greater (see Figs. 6-7). 


\section{References}

[1] ROSFOND-2010 Library, Institute of Physics and Power Engineering, Obninsk, Russia (2010). Available online at http://www.ippe.ru/podr/abbn/libr/rosfond.php.

[2] K. Shibata et al., "JENDL-4.0: A new library for nuclear science and engineering” J. Nucl. Sci. Technol.. 48, pp. 1-30, 2011.

[3] M.B. Chadwick et al., "ENDF/B-VII.1 Nuclear data for science and technology: cross sections, covariances, fission product yields and decay data," Nuclear Data Sheets, v.112, 12, 2011

[4] The JEFF-3.1.1 Nuclear Data Library, JEFF Report 22, NEA No. 6807, OECD 2009.

[5] S.F. Mughabghab, "Atlas of Neutron Resonances: Thermal Cross Sections and Resonance Parameters", Elsevier Publisher, Amsterdam, 2006.

[6] Experimental Nuclear Reaction Data (EXFOR / CSISRS), http://www.nndc.bnl.gov/exfor/exfor00.htm

[7] International Handbook of Evaluated Criticality Safety Benchmark Experiments, OECD/NEA, NEA/NSC/DOC(95)03 (September 2009 Edition)

[8] Yu.E. Golovko, T.T. Ivanova, M.N. Nikilaev et al., VANT, Nuclear Constants, v. 1-2, p.110-125, (2007).

[9] Norman L. Pruvost, Hugh C. Paxton, Nuclear Criticality Safety Guide./ LA-12808, UC-714, (1996)

[10] R.E. MacFarlane et al. NJOY97.0 Code System for Produsing Pointwise and Multigroup Neutron and Photon Sections from ENDF/B Data. RSIC Peripheral Shielding Routine Collection, PSR-368.

[11] Bethe N.A., Beyster J.R., Carter R.E. Inelastic Cross Sections for Fission Spectrum Neutrons IV, J.Nucl.Energy, 1957, v.4, p,147 (EXFOR SUBENT 11461000).

[12 ] B.B. Forrest, T.E. Booth et al. "MCNP-A General Monte Carlo N-Particle Transport Code, Version 5, Overview and Theory, Volume I", LA-UR-03-1987, LANL, (2003) 